\title{
A Solution of the Cosmological Constant and DE and Arrow of Time, Using Model of a Nonsingular Universe from Rosen from Volume (56) Ettore Majorana International Science Series, Physics, 1991
}

\author{
Andrew Beckwith \\ Physics Department, College of Physics, Chongqing University, Chongqing, China \\ Email: rwill9955b@gmai.com
}

How to cite this paper: Beckwith, A. (2021) A Solution of the Cosmological Constant and DE and Arrow of Time, Using Model of a Nonsingular Universe from Rosen from Volume (56) Ettore Majorana International Science Series, Physics, 1991. Journal of High Energy Physics, Gravitation and Cosmology, 7, 925-935.

https://doi.org/10.4236/jhepgc.2021.73053

Received: March 31, 2021

Accepted: June 28, 2021

Published: July 1, 2021

\section{Copyright $\odot 2021$ by author(s) and} Scientific Research Publishing Inc. This work is licensed under the Creative Commons Attribution International License (CC BY 4.0).

http://creativecommons.org/licenses/by/4.0/

\section{(cc) (i) Open Access}

\begin{abstract}
We reduplicate the Book "Dark Energy" by M. Li, X-D. Li, and Y. Wang, given zero-point energy calculation with an unexpected "length" added to the "width" of a graviton wave just prior to specifying the creation of "gravitons", using the Rosen and Israelit model of a nonsingular universe. In doing so we are in addition to obtaining a wavelength $10^{30}$ times greater than Planck's length so we can calculate DE, may be able to with the help of the Rosen and Israelit model have a first approximation as to the arrow of time, and a universe with massive gravity. We have left the particulars of the nonsingular starting point undefined but state that the Rosen and Israelit model postulates initial temperatures of $10^{-180}$ Kelvin and also a value of about Planck temperature, at $10^{-3}$ centimeters radii value which may satisfy initial conditions asked by t'Hooft for describing an arrow of time. A key assumption is that the $\mathrm{DE}$ is formed at $10^{-3} \mathrm{~cm}$, after an expansion of $10^{30}$ times in radii, from the Planck length radius nonsingular starting point. The given starting point for $\mathrm{DE}$ in this set of assumptions is where there is a change in the cosmic acceleration, to a zero value, according to Rosen and Israel, with time $t=1.31$ times $10^{-42}$ seconds. That may be where we may specify a potential magnitude, $V$, which has ties into inflaton physics. The particulars of the model from Rosen and Israelit allow a solution to be found, without discussion of where that nonsingular starting point came from, a point the author found in need of drastic remedies and fixes.
\end{abstract}

\section{Keywords}

Minimum Scale Factor, Cosmological Constant, Space-Time Bubble, DE, 


\section{Introduction}

What we are doing is to try to confirm if we can apply the techniques of the following reference to the problem of DE and the arrow of time, and heavy gravity. After work I did in [1] was allegedly not credible, due to people having doubts as to the existence of a multiverse and equating two first integrals as I did, via early pre Planckian space-time, the following reference was accessed [2], and then was applied to [3] and the work on heavy gravity in [4]. In doing so we will keep in mind the 't Hooft memorandum as to the arrow of time, which is in [5] as a basic organizational principle for our discussion, i.e. formation of our program is assuming initial conditions for using [4] in the expansion of the universe say after $10^{-42}$ seconds.

$$
m_{g}=\frac{\hbar \sqrt{\Lambda}}{c}
$$

Whereas we ask for initial conditions for the arrow of time, and $\Lambda$ and DE formation.

\section{Methods for Defining DE and Heavy Gravity}

We will first start off with the redone calculation as to the Vacuum energy as given in [3] and how we rescale them to be in sync as to the observed experimentally given value for vacuum energy which is of the present era. This methodology is consistent with the Zero-point energy calculation, we start off with the following as given by [3].

$$
\begin{aligned}
& \frac{1}{2} \cdot \sum_{i} \omega_{i} \equiv V(\text { volume }) \cdot \int_{0}^{\lambda} \sqrt{k^{2}+m^{2}} \frac{k^{2} \mathrm{~d} k}{4 \pi^{2}} \approx \frac{\bar{\lambda}^{4}}{16 \pi^{2}} \\
& \underset{\hat{\lambda}=M_{\text {Planck }}}{\longrightarrow} \rho_{\text {boson }} \approx 2 \times 10^{71} \mathrm{GeV}^{4} \approx 10^{119} \cdot\left(\rho_{\mathrm{DE}}=\frac{\Lambda}{8 \pi G}\right)
\end{aligned}
$$

In stating this we have to consider that $\rho_{\mathrm{DE}}=\frac{\Lambda}{8 \pi G} \approx \hbar \cdot \frac{(2 \pi)^{4}}{\lambda_{\mathrm{DE}}^{4}}$, so then that the equation we have to consider is a wavelength $\lambda_{\mathrm{DE}} \approx 10^{30} \ell_{\text {Planck }}$ which is about $10^{30}$ times a Plank length radius of a space-time bubble. That would, we have after $10^{-42}$ seconds.

$$
\lambda_{\mathrm{DE}} \approx 10^{30} \ell_{\text {Planck }}
$$

We then have to consider how to reach the experimental conditions for when,

$$
\rho_{D E}=\frac{\Lambda}{8 \pi G} \approx \hbar \cdot \frac{(2 \pi)^{4}}{\lambda_{\mathrm{DE}}^{4}}
$$

A nonsingular expansion point for cosmology will after $10^{-42}$ seconds lead to Equation (4). That means a discussion of what Rosen and Israelit did in [2]. Our point 
applying [2] to Equation (3) is that we have a factor of $10^{30}$ expansion as to where we can at least measure the onset of $\mathrm{DE}$, for reasons which will be in the next section so Equation (4) has a value of roughly DE in magnitude as given in [3].

\subsection{Looking Now at Rosen and Israelit, in Terms of Thermodynamics of Anon-Singular Universe Will Be Relevant for Several Reasons}

1) We will be able to come up with an initial temperature of $10^{-180}$ Kelvin, at about Planck length, in value. Almost absolute zero;

2) The temperature of space-time will be of the order of Planck Temperature after expansion of about $10^{30}$ times from the initial nonsingular configuration;

3) For making effective use of [3] we will be looking at Equation (1) to Equation (4) as being measured after $10^{-42}$ seconds, which is roughly Planck Time, in this model, i.e., the convention is that we will be using is that Equation (1) to Equation (4) will be what [2] calls the pre-matter radiation transition point, in the history of the universe, i.e., go to pages 153 to 154, of [2] and one sees that what I am doing is specifying the formation of Equation (1) to Equation (4) at the time the acceleration of the universe stops in its earliest phase, with the formation of DE, and cosmological Constant. We should also keep in mind that $\mathrm{A}$. and $\mathrm{B}$. and $\mathrm{C}$ will allow an arrow of time forming due to the reasons brought up in [5] whereas we have the following Entropy value of [6].

$$
S \sim 3 \cdot\left[1.66 \sqrt{g_{*}}\right]^{2} T^{3}
$$

whereas we have that [7] gives us a value $g_{*} \approx 100-110$. Hence it is time to do the treatment of the temperature values, of what that says about Entropy, and the arrow of time.

\subsection{Underlying Thermodynamics of the Rosen-Israelit Nonsingular Model}

In this section we outline temperature values $\mathrm{T}$ at beginning of expansion, at the end of expansion up to when DE is formed and answer if [5] criteria as to forming the arrow of time can be formed according to [5]. While noting the issue of casuality and causal relations, in the context of the arrow of time [8] where we take into consideration the following, namely that,

Quote:

The causal sets program [8] is an approach to quantum gravity. Its founding principles are spacetime is fundamentally discrete (a collection of discrete spacetime points, called the elements of the causal set) and that spacetime events are related by a partial order. This partial order has the physical meaning of the causality relations between spacetime events.

End of quote:

What we will assert is that the Rosen result, given in [2] may permit the introduction of the partial order in space-time which may allow for the introduction of quantum gravity. 


\subsection{Formal Development of the Thermodynamics of Space-Time and Its to DE}

The key point of this mini chapter will be to summarize the derivation of the temperature [2].

$$
T=\left(\rho_{P} / \sigma\right)^{1 / 4} \cdot \frac{\breve{a} r^{7}}{\left(\breve{a}^{4}+r^{4}\right)^{2}}
$$

whereas $\left(\rho_{P} / \sigma\right)^{1 / 4}=1.574 \times 10^{32} \mathrm{~K}$ (kelvin) , and $\breve{a}=10^{-3} \mathrm{~cm}$, whereas,

$$
\begin{aligned}
& r_{\text {initial }}=\left(3 / 8 \pi \rho_{P}\right)^{1 / 2}=5.58 \times 10^{-34} \mathrm{~cm} \\
& \Rightarrow T_{\text {initial }}=2.65 \times 10^{-180} \mathrm{~K}(\text { kelvin }) \\
& r_{\text {DE-formation }}=\breve{a}=10^{-3} \mathrm{~cm} \\
& \Rightarrow T_{\text {DE-formation }}=7.41 \times 10^{31} \mathrm{~K}(\text { kelvin })
\end{aligned}
$$

We will be deriving Equation (6) as a summary of what to expect in this treatment of nonsingular space-time.

To do so we start off with [2] in pre matter and radiation periods with entropy $\mathcal{S}, \rho=\rho(T), P=P(T)$,

$$
\begin{gathered}
\mathrm{d} S(V, T)=\frac{1}{T} \cdot[\mathrm{d}(\rho V)+P \mathrm{~d} V] \\
V=V(\text { volume })=2 \pi^{2} r^{3}
\end{gathered}
$$

And an integratability condition on Equation (6) leading to:

$$
\frac{\mathrm{d} P}{\mathrm{~d} T}=\frac{1}{T} \cdot(\rho+P)
$$

Then the integral of Equation (7) is given as:

$$
S=\frac{V}{T} \cdot(\rho+P)
$$

Also, we look at a given value of pressure as given in [2] for which:

$$
P=\frac{\rho}{3} \cdot\left(1-\frac{4 \rho}{\rho_{P}}\right)
$$

Put Equation (7d) into Equation (7b) and then one will get after integrating Equation (7b),

$$
\rho \cdot\left(1-\frac{\rho}{\rho_{P}}\right)^{7}=\sigma T^{4}
$$

Here, [2] treated $\sigma$ as the Stephan-Boltzman constant, and so then if we add in the energy equation,

$$
\dot{\rho}+3 \cdot(\dot{r} / r) \cdot(\rho+P)=0
$$

Then we put in Equation (8) into Equation Equation (8b) we obtain:

$$
\rho=\breve{a}^{4} \rho_{P} /\left(\breve{a}^{4}+r^{4}\right)
$$

We claim that Equation (8c) put into Equation (8a) we wll then obtain Equa- 
tion (6) with the conditions as we specified. We assert that we obtain through Equation (6), Equation (6a) and Equation (6b) when the temperature is in the vicinity Planck temperature, that then we can introduce conditions for which we have Equation (4) implemented [3] we have a value of Planck's constant is at the value given in Equation (6b) which [2] is the prematter radiation boundary, so then that we are initiating $\mathrm{DE}$ as a function as the onset of the radiation era of cosmology, and when DE commences we have by Equation (1) conditons for the onset of gravitational physics.

\subsection{Analysis of the Interrelationship between Terms in the Inflaton, for Cosmology, Inhomogeniety and Temperature $T$, at the Prematter-Radition Boundary}

We are going to use the following result from [9] of:

$$
\frac{H^{2}}{\dot{\phi}} \approx 10^{-5}
$$

Whereas, we are using by [10], page 481 of that reference,

$$
H=1.66 \sqrt{g_{*}} \cdot \frac{T_{\text {temp }}^{2}}{m_{P}}
$$

Whereas we have from [11] the following time derivative value of the inflaton leading to, if we use Equation (9) and also Equation (9a),

$$
\begin{aligned}
& a(t)=a_{\text {initial }} t^{\nu} \\
& \Rightarrow \phi=\ln \left(\sqrt{\frac{8 \pi G V_{0}}{v \cdot(3 v-1)}} \cdot t\right) \sqrt{\frac{v}{16 \pi G}} \\
& \Rightarrow \dot{\phi}=\sqrt{\frac{v}{4 \pi G}} \cdot t^{-1} \\
& \Rightarrow \frac{H^{2}}{\dot{\phi}} \approx \sqrt{\frac{4 \pi G}{v}} \cdot t \cdot T^{4} \cdot \frac{(1.66)^{2} \cdot g_{*}}{m_{P}^{2}} \approx 10^{-5}
\end{aligned}
$$

If we make use of Planck units, for $t \sim$ Planck time, $G=1$, and Planck mass set $=1$, and Planck Temperature $T$ set also to 1 then Equation (10) says that the coefficient $v$ just before turnabout, i.e. where the acceleration of inflation stopped is still very large, but not infinite, whereas if we do not do such Planck units, the terms $\mathrm{t}$ times $T$, representing time, $t$, and the $4^{\text {th }}$ power of temperature $T$, mean that if we have, indeed nearly Plank temperatures, for $T$, that the time element $t$ would be very small and so verifying the largeness of coefficient $v$ just before we have a cessation of acceleration, initially.

If instead of using Equation (9) for $H$, we use instead from [11] the following value of $H$ as given in [11] only,

$$
H^{2}=V_{0} \exp \left(-\sqrt{\frac{16 \pi G}{v}} \phi\right)=V_{0} \cdot\left(\sqrt{\frac{8 \pi G V_{0}}{v \cdot(3 v-1)}} \cdot t\right)^{\frac{1}{2} \cdot \sqrt{\frac{v}{\pi G}}-4 \sqrt{\frac{\pi G}{v}}}
$$

We then will get: 


$$
\frac{H^{2}}{\dot{\phi}} \approx \sqrt{\frac{4 \pi G}{v}} \cdot V_{0} \cdot\left(\sqrt{\frac{8 \pi G V_{0}}{v \cdot(3 v-1)}}\right)^{\frac{1}{2} \cdot \sqrt{\frac{v}{\pi G}}}-4 \sqrt{\frac{\pi G}{v}} t^{\frac{1}{2} \cdot \sqrt{\frac{v}{\pi G}}-4 \sqrt{\frac{\pi G}{v}}+1} \approx 10^{-5}
$$

If we wish to just focus upon a general value for this $10^{-5}$ value, semi independent of time we can:

set,

$$
\frac{1}{2} \cdot \sqrt{\frac{v}{\pi G}}-4 \sqrt{\frac{\pi G}{v}}+1=0
$$

A particular solution if we look at Planck units for which $G=1$ is $v=5$ which would then putvery precise conditions upon $V_{0}$, I.e. in Planckian units with $G=$ 1 we would have in Plank units, normalized to $=1$,

$$
\sqrt{\frac{4 \pi}{5}} \cdot V_{0} \cdot\left(\sqrt{\frac{8 \pi V_{0}}{5 \cdot 14}}\right)^{\frac{1}{2} \cdot \sqrt{\frac{5}{\pi}}-4 \sqrt{\frac{\pi}{5}}} \approx 10^{-5}
$$

Note this is a particular solution but it would serve to put in approximate values for $V_{0}$ about the time would have the formation of DE, and the cosmological constant, at a time step value approximately $10^{-42}$ seconds, at the time we have the first case of when the velocity would be maximized in inflation at the boundary of pre-matter and radiation, to quote [2].

\subsection{What Does Equation (5) Tell Us about the Arrow of Time, Problem? Using [2]?}

Were this to be true and the near zero temperature as given by Equation (6a) versus the near Planck temperature at Equation (6b), then going to the entropy expression of Equation (5), we do have in this situation matching the requirements given by ' $t$ Hooft, [5] for which we can state that the construction of Equation (5) combined with ' $t$ Hooft's particular solutions for initial conditions to the arrow of time, may indeed give a consistent arrow of time solution.

\subsection{What about the Matter of Causal Relations and Initial Conditions, Using Dowker's Construction and Discussion of Posets?}

The author in [12] had this initial construction, i.e., and is replicated for the record with several given Changes. We first give initial equations of [7] [13] and then after words relate it to the Dowker [8] results Here, the idea would be, to make the following equivalence, i.e., look at, [7] where we have what we call Initial entropy value for when we identify the cosmological constant. The value of Equation (12) is assumed to be in magnitude about $10^{90}$ or so, which is the value of entropy if we use the following sort of model:

$$
\left[\left[\frac{\Lambda_{M a x} r^{4}}{8 \pi G}\right] \cdot 4 / 3 \cdot\left[\frac{2 \pi^{2} g_{*}}{45}\right]^{1 / 3}\right]^{3 / 4} \sim S_{\text {initial }}
$$

We furthermore, make the assumption of a minimum radius of [14] [15] 
where the $r$ in Equation (12) is the same in Equation (13) below, and in magnitude $10^{30}$ times larger than when Entropy was effectively zero.

$$
R_{\text {initial }}(\text { when } \Lambda \text { forms })=\text { radius is } 10^{30} \text { Planck length }
$$

This Equation (12) will be put as the minimum value of $r$, where we have in this situation [16] [17] with $M$ the amount. Space-time matter energy at the start of the radiation era, and $l \approx 10^{29}-10^{30}$ start radius as given in Equation (6b), with the start radius when we have almost zero entropy. If so then we have at $10^{-3}$ centimeters,

$$
\text { \# bits } \sim\left[\frac{E}{\hbar} \cdot \frac{l}{c}\right]^{3 / 4} \approx\left[\frac{M c^{2}}{\hbar} \cdot \frac{l}{c}\right]^{3 / 4}
$$

Needless to say we would have entropy defined as Equation (14) to the $4 / 3^{\text {rd }}$ power, as to have a linkage between Entropy, bits and also the grid points in a space-time lattice which may give us quantum gravity. Afterwards we likely to keep fidelity with the results we have worked with prior to this section have an invariant cosmological constant and would be applying our inquiry as to the application of Equation (12) as of where the cosmological constant formed up in an identifiable manner. Meaning, after $10^{-42}$ seconds, and at a radius of $10^{-3}$ centimeters, in line with the mass $M$ being the "equivalent matter energy" at the boundary between pre matter states, and radiation as given in [2]. Keep in mind that the Energy $E$ as given in Equation (14) would have temperature dependence as given in Equation (6b) with an input parameter of $E$ which can go into Equation (14),

$$
E=\frac{k_{B}(\operatorname{dim} \text { space time })}{2} \cdot T
$$

The points where we have bits, as computationally given would be the grid points to the Poset argument as in [8]. Whereas we can give the following relationship to specify the inter-relationship between $E$ and time. We pick Entropy as represented by an energy term $E$ driving the entropy as given by $T$ temperature dependence. As given in Equation (15), whereas we use the cube of the same Temperature $T$ driving entropy in Equation (12).

\subsection{Coming Up with a "Modified HUP" So As to Obtain the Grid Points Implied by Equation (14)}

Shalyt-Margolin and Tregubovich [18], Shalyt-Margolin [19] [20] have this relationship. Here delta $E$ is assumed to be consistent in a change in energy from almost zero to the energy value give in Equation (15),

$$
\begin{aligned}
& \Delta t \geq \frac{\hbar}{\Delta E}+\gamma t_{P}^{2} \frac{\Delta E}{\hbar} \Rightarrow(\Delta E)^{2}-\frac{\hbar \Delta t}{\gamma t_{P}^{2}}(\Delta E)^{1}+\frac{\hbar^{2}}{\gamma t_{P}^{2}}=0 \\
& \Rightarrow \Delta E=\frac{\hbar \Delta t}{2 \gamma t_{P}^{2}} \cdot\left(1+\sqrt{1-\frac{4 \hbar^{2}}{\gamma t_{P}^{2} \cdot\left(\frac{\hbar \Delta t}{2 \gamma t_{P}^{2}}\right)^{2}}}\right)=\frac{\hbar \Delta t}{2 \gamma t_{P}^{2}} \cdot\left(1 \pm \sqrt{1-\frac{16 \hbar^{2} \gamma t_{P}^{2}}{(\hbar \Delta t)^{2}}}\right)
\end{aligned}
$$


For sufficiently small $\gamma$. The above could be represented by [20],

$$
\begin{aligned}
& \Delta E \approx \frac{\hbar \Delta t}{2 \gamma t_{P}^{2}} \cdot\left(1 \pm\left(1-\frac{8 \hbar^{2} \gamma t_{P}^{2}}{(\hbar \Delta t)^{2}}\right)\right) \\
& \Rightarrow \Delta E \approx \text { either } \frac{\hbar \Delta t}{2 \gamma t_{P}^{2}} \cdot \frac{8 \hbar^{2} \gamma t_{P}^{2}}{(\hbar \Delta t)^{2}}, \text { or } \frac{\hbar \Delta t}{2 \gamma t_{P}^{2}} \cdot\left(2-\frac{8 \hbar^{2} \gamma t_{P}^{2}}{(\hbar \Delta t)^{2}}\right)
\end{aligned}
$$

This would lead to a minimal relationship between change in $E$ and change in time as represented by Equation (17), so that we could to first order, say be looking at something very close to the traditional Heisenberg uncertainty principle results of approximately,

$$
\Delta E \approx \frac{\hbar \Delta t}{2 \gamma t_{P}^{2}} \cdot \frac{8 \hbar^{2} \gamma t_{P}^{2}}{(\hbar \Delta t)^{2}} \equiv \frac{4 \hbar}{\Delta t}
$$

Or,

$$
\Delta E \Delta t \approx 4 \hbar
$$

i.e., stepping through an iteration of delta $t$, per causal structure in early space-time would with refinements help construct the grid and causal structures alluded to in [8], in particular see her discussion of the causal set given in page 3 of [8] whereas the grid defined by [8] would reflect the computational "bits" given in Equation (14).

\section{Good Points and Limitations as to the Given Analysis, and What Needs to Be Filled In}

Our analysis has given evidence that we can satisfy the ' $t$ Hoof idea of special initial conditions as to forming the arrow of time. This is important, since there is a basic symmetry in the GR equations of space-time, which means forming the arrow of time, will necessitate specialized initial condtions even if the general GR equations do not depend upon specialized initial conditions. In addition, in the face of virtually unaminous complaints on the part of reviewers, the author has avoided describing the parcicular origins of a non singular start to expansion of the universe. The Rosen and Israel model assumes this nonsingular start, as seen in [2], without trying to derive where it came from. The author states that [2] gives a thermodynamically consistent nonsingular univese model which satisfied a mathematically consistent origin to the arrow of time problem. Due to the quirkiness of the [2] model, Rosen and Israelit also called the start of this expansion as a Point of zero time. That is right. The start to expansion is called time value "zero". The unusal nature of this designation allowed the author to then go to a minimum time step, delta $t$ which may be measurable, if one obtains in data sets a boundary regime which delinates the start of the radiation regime in cosmology.

Now for the limitations,

The author abandoned any attemp in this document to specify where the nonsingular start to cosmological expansion came from. As a physics researcher, this 
is an appalling omission, and is only done due to the innate conservatism of the general research community. What is mandatory is that aderivational approach to the origins of this nonsingular start be somehow meshed into a research program of hopefully gravitational physics data sets. In addition, [21] in terms of holographic principle applications of an interrelationhip between the mass of a gravition and information needs to be explored in lieu of specifying the time of delta $t$ approximately $10^{-42}$ seconds and a defined initial space-time the following was obtained in terms of probable GW signals, from this early universe configuration.

\section{How Do We Obtain Relic High Frequency Gravity Waves?}

With redshift about $z=10^{25}$ we go work with the following approximation:

$$
\begin{aligned}
& \left(1+z_{\text {initial era }}\right) \equiv \frac{a_{\text {today }}}{a_{\text {initial era }}} \approx\left(\frac{\omega_{\text {Earth orbit }}}{\omega_{\text {initial era }}}\right)^{-1} \\
& \Rightarrow\left(1+z_{\text {initial era }}\right) \omega_{\text {Earth orbit }} \approx 10^{25} \omega_{\text {Earth orbit }} \approx \omega_{\text {initial era }}
\end{aligned}
$$

We postulate that we specify an initial era frequency via dimensional analysis which is slightly modified by Maggiore for the speed of a graviton [22] whereas we use that we assume having the following relationship of

$c($ light speed $) \approx \omega_{\text {initial era }} \cdot\left(\lambda_{\text {initial post bubble }}=\ell_{\text {Planck }}\right)$ and that dimensional comparison with initially having a temperature built up so as $\Delta E \approx \hbar \omega_{\text {initial era }}$ where $T_{\text {universe }} \approx T_{\text {Plank temerature }}=1.22 \times 10^{19} \mathrm{GeV}$.

If so then the initial temperature would be extremely high leading to a change in temperature from Pre Planckian conditions to Planck era. Where we would be assuming $\omega_{\text {initial era }} \approx \frac{c}{\ell_{\text {planck }}} \leq 1.8549 \times 10^{43} \mathrm{~Hz}$ so then we would be looking at having frequencies on Earth from gravitons of mass m (graviton) less than of equal to:

$$
\omega_{\text {Earth orbit }} \leq 10^{-25} \omega_{\text {initial era }}
$$

This is what would necessitate new technological developments and likely space-borne systems to analyze the final point being that the brilliant work done by Rosen in [24] needs to be explored as possibly being relevant to the origins of the nonsingular start to the cosmological expansion. The author views [23] as a worthy starting point to quantum mechanical analogues which may explain this datum, missing in the [2] nonsingular start to the present universe which should be explored, as to its relevance to quantum mechanics and near space-time singularities. Rosen's [23] model may provide a bridge between interior conditions which may exist in a nonsingular start to expansion of the universe, and what is happening in our present cosmos. That as doing generalizations of what $\mathrm{Ng}$ [17] proposed as far as infinite quantum statistics for a counting algorithm approach to early universe entropy. We also view that what is presented in [24] as to a quantum vacuum will be decisively important to explain the transition from the pre-inflationary state, as implied by [2] and the rapid expansion as given in Equation (6), Equation (6a) and Equation (6b). 


\section{Acknowledgements}

This work is supported in part by National Nature Science Foundation of China grant No. 11375279.

\section{Conflicts of Interest}

The author declares no conflicts of interest regarding the publication of this paper.

\section{References}

[1] Beckwith, A.W. (2021) Using "Enhanced Quantization" to Bound the Cosmological Constant, (for a Bound-on Graviton Mass), by Comparing Two Action Integrals (One Being from General Relativity) at the Start of Inflation. In: Sidharth, B.G., Murillo, J.C., Michelini, M. and Perea, C., Eds., Fundamental Physics and Physics Education Research, Springer Nature Switzerland AG, Gewerbestrasse, Switzerland, 21-35. https://doi.org/10.1007/978-3-030-52923-9_3

[2] Rosen, N. and Israelit, M. (1991) A Simple Model of the Universe without Singularities. In: Zichichi, A., de Sabbata, V. and Sánchez, N., Eds., Gravitation and Modern Cosmology, Ettore Majorana International Science Series, Vol 56, Springer, Boston, MA. https://doi.org/10.1007/978-1-4899-0620-5_14

[3] Li, M., Li, X.-D., Wang, S. and Wang, Y. (2015) Dark Energy. Peking UniversityWorld Scientific, Singapore, Republic of Singapore.

[4] Novello, M. (2005) The Mass of the Graviton and the Cosmological Constant Puzzle. https://arxiv.org/abs/astro-ph/0504505

[5] 't Hooft, G. (2018) Time, the Arrow of time and Quantum Mechanics. Frontiers in Physics, 6, Article No. 81. https://doi.org/10.3389/fphy.2018.00081

[6] Beckwith, A.W. (2018) Initial Conditions for Defining an Arrow of Time at the Start of Inflation? Journal of High Energy Physics. Gravitation and Cosmology, 4, 787-795. https://doi.org/10.4236/jhepgc.2018.44044

[7] Kolb, E. and Turner, S. (1994) The Early Universe. Westview Press, Chicago, USA.

[8] Fay, D. (2005) Causal Sets and the Deep Structure of Space-Time. https://arxiv.org/abs/gr-qc/0508109

[9] Sergi, W. (2009) Eternal Inflation. World Scientific Publishing Co., Pte. Ltd. Singapore, the Republic of Singapore. https://doi.org/10.1142/6923

[10] Utpal, S. (2008) Particle and Astroparticle Physics. Taylor \& Francis Group, New York City, New York, USA.

[11] Thanu, P. (2006) World Scientific Series in Astronomy and Astrophysics: Volume 8, Singapore: An Invitation to Astrophysics. World Press Scientific, Republic of Singapore. https://doi.org/10.1142/6010

[12] Beckwith, A.W. (2018) How a Minimum Time Step and Formation of Initial Causal Structure in Space-Time May Void the Penrose Singularity Theorem, as in Hawkings and Ellis's 1973 Write-Ups. Journal of High Energy Physics, Gravitation and Cosmology, 4, 485-491. https://doi.org/10.4236/jhepgc.2018.43026

[13] Mukhanov, V. (2005) Physical Foundations of Cosmology. Cambridge University Press, New York, USA. https://doi.org/10.1017/CBO9780511790553

[14] Camara, C.S., de Garcia Maia, M.R., Carvalho, J.C. and Lima, J.A.S. (2004) Nonsingular FRW Cosmology and Non Linear Dynamics. Physical Review D, 69, Article ID: 123504. https://doi.org/10.1103/PhysRevD.69.123504 
[15] Beckwith, A. (2016) Gedanken Experiment for Refining the Unruh Metric Tensor Uncertainty Principle via Schwarzschild Geometry and Planckian Space-Time with Initial Nonzero Entropy and Applying the Riemannian-Penrose Inequality and Initial Kinetic Energy for a Lower Bound to Graviton Mass (Massive Gravity). Journal of High Energy Physics, Gravitation and Cosmology, 2, 106-124. https://doi.org/10.4236/jhepgc.2016.21012

[16] Park, D., Kim, H. and Tamarayan, S. (2002) Nonvanishing Cosmological Constant of Flat Universe in Brane-World Scenario. Physics Letters B, 535, 5-10. https://doi.org/10.1016/S0370-2693(02)01729-X

[17] Ng, Y.J. (2007) Holographic Foam, Dark Energy and Infinite Statistics. Physics Letters $B, 657,10-14$. https://doi.org/10.1016/j.physletb.2007.09.052

[18] Shalyt-Margolin, A.E. (2006) Deformed Density Matrix and 21 Quantum Entropy of the Black Hole. Entropy, 8, 31-43. https://doi.org/10.3390/e8010031

[19] Shalyt-Margolin, A.E. (2005) The Density Matrix Deformation in Physics of the Early Universe and Some of its Implications. Quantum Cosmology Research Trends. Horizons in World Physics, 246, 49-91.

[20] Shalyt-Margolin, A.E., Tregubovich, A.Y. (2004) Deformed Density Matrix and Generalized Uncertainty Relation in Thermodynamics. Modern Physics Letters A, 19, 71-81. https://doi.org/10.1142/S0217732304012812

[21] Haranas I. and Gkigkitzis, I. (2014) The Mass of Graviton and Its Relation to the Number of Information according to the Holographic Principle. International Scholarly Research Notices, 2014, Article ID: 718251. https://doi.org/10.1155/2014/718251

[22] Michele, M. (2008) Gravitational Waves, Volume 1, Theory and Experiment. Oxford University Press, New York, USA.

[23] Rosen, N. (1993) Quantum Mechanics of a Miniuniverse. International Journal of Theoretical Physics, 32, 1435-1440. https://doi.org/10.1007/BF00675204

[24] Wang, Q.d., Zhen, Z. and Unruh, W.G. (2017) How the Huge Energy of Quantum Vacuum Gravitates to Drive the Slow Accelerating Expansion of the Universe. Physical Review D, 95, Article ID: 103504. https://doi.org/10.1103/PhysRevD.95.103504 